\title{
Potential role of nuclear magnetic resonance spectroscopy to identify salivary metabolite alterations in patients with head and neck cancer
}

\author{
JOPI J.W. MIKKONEN ${ }^{1,2}$, SURYA P. SINGH ${ }^{2,3}$, RAMIN AKHI $^{4}$, \\ TUULA SALO $^{5-8}$, REIJO LAPPALAINEN ${ }^{1,9}$, WILFREDO A. GONZÁLEZ-ARRIAGADA ${ }^{10}$, \\ MÁRCIO AJUDARTE LOPES ${ }^{11}$, ARJA M. KULLAA ${ }^{2,4,12}$ and SAMI MYLLYMAA ${ }^{1,9}$ \\ ${ }^{1}$ SIB Labs; ${ }^{2}$ Institute of Dentistry, University of Eastern Finland, FI-70211 Kuopio, Finland; \\ ${ }^{3}$ Laser Biomedical Research Centre, Massachusetts Institute of Technology, Boston, MA 02139, USA; \\ ${ }^{4}$ Research Unit of Oral Health Sciences; ${ }^{5}$ Cancer and Translational Medicine Research Unit, University of Oulu; \\ ${ }^{6}$ Medical Research Center, Oulu University Hospital, FI-90014 Oulu; ${ }^{7}$ Department of Oral and Maxillofacial Diseases, \\ University of Helsinki; ${ }^{8}$ HUSLAB, Helsinki University Hospital, FI-00014 Helsinki; ${ }^{9}$ Department of Applied Physics, \\ University of Eastern Finland, FI-70211 Kuopio, Finland; ${ }^{10}$ Oral Pathology and Diagnosis, School of Dentistry, \\ Universidad de Valparaiso, Valparaiso, Región de Valparaíso 2360004, Chile; ${ }^{11}$ Department of Oral Diagnosis, \\ School of Dentistry, State University of Campinas, Sao Paulo CEP 13414-018, Brazil; \\ ${ }^{12}$ Educational Dental Clinic, Kuopio University Hospital, FI-70029 Kuopio, Finland
}

Received March 5, 2018; Accepted August 30, 2018

DOI: 10.3892/ol.2018.9419

\begin{abstract}
The analysis of the salivary metabolomic profile may offer an early phase approach to assess the changes associated with a wide range of diseases including head and neck cancer. The aim of the present study was to investigate the potential of nuclear magnetic resonance (NMR) spectroscopy for detecting the salivary metabolic changes associated with head and neck squamous cell carcinoma (HNSCC). Unstimulated whole-mouth saliva samples collected from HNSCC patients (primary tumour was located either in the larynx or in the oral cavity) and healthy controls were analysed by ${ }^{1} \mathrm{H}-\mathrm{NMR}$ spectroscopy. Reliably identified salivary metabolites were quantified and the determined concentration values were compared group-wise using a Mann-Whitney U-test. Multivariate discrimination function analysis (DFA) was conducted to identify such a combination of metabolites, when considered together, that gives maximum discrimination between the groups. HNSCC patients exhibited significantly increased concentrations of 1,2-propanediol $(\mathrm{P}=0.032)$ and fucose $(\mathrm{P}=0.003)$, while proline levels were significantly decreased $(\mathrm{P}=0.043)$. In the DFA model, the most powerful
\end{abstract}

Correspondence to: Dr Sami Myllymaa, Department of Applied Physics, University of Eastern Finland, PO Box 1627, FI-70211 Kuopio, Finland

E-mail: sami.myllymaa@uef.fi

Key words: metabolomics, saliva, squamous cell carcinoma, nuclear magnetic resonance spectroscopy, biomarkers discrimination was achieved when fucose, glycine, methanol and proline were considered as combined biomarkers, resulting in a correct classification rate of $92.1 \%$, sensitivity of $87.5 \%$ and specificity of $93.3 \%$. To conclude, NMR spectrometric analysis was revealed to be a feasible approach to study the metabolome of saliva that is sensitive to metabolic changes in HNSCC and straightforward to collect in a non-invasive manner. Salivary fucose was of particular interest and therefore, controlled longitudinal studies are required to assess its clinical relevance as a diagnostic biomarker in HNSCC.

\section{Introduction}

Head and Neck Squamous Cell Carcinoma (HNSCC) is one of the most common cancers and accounts for $4 \%$ of total malignant tumours worldwide (1). HNSCC includes tumours of the various sites of upper aerodigestive tract including the pharynx, larynx, sinuses, nasal cavity and oral cavity. HNSCC is an aggressive disease and associated with high mortality and morbidity rates that are mainly attributed to the late detection. Lack of reliable biomarkers and simple and accurate diagnostic tools for the screening of early stage cancers are the major obstacles to hurdles in reducing the mortality rates (2).

Saliva is known to be capable of mirroring the status of both oral and systemic health (3). It contains locally expressed proteins and end-products of different metabolic pathways (i.e., metabolites) that are known to alter greatly in their concentrations in various diseases (4). Therefore, these substances, called as salivary biomarkers, are good indicators of an individual's health status. Over the last years, considerable efforts have been made to clarify the potential of salivary metabolomics as an alternative diagnostic tool (3-6). Salivary metabolites 
are powerful in elucidating the pathways underlying different diseases and thereby they can be considered as ideal for the early diagnostics of various diseases, such as HNSCC (7-9). The use of salivary biomarkers is especially attractive in oral cancer, since the tumours communicate with saliva. Furthermore, tumour-derived extracellular vesicles might lead to the development of tumour-specific salivary biomarkers (10-13).

Nuclear magnetic resonance (NMR) spectroscopy is a quantitative technique based on the magnetic properties of atomic nuclei. When the sample is placed in an external magnetic field, NMR active nuclei (e.g., ${ }^{1} \mathrm{H}$ and $1{ }^{3} \mathrm{C}$ ) absorb electromagnetic radiation and move from a low-energy spin stage to a high-energy spin stage (14). When exposed with radiofrequency pulses, the nuclei emit electromagnetic radiation and move back to a low-energy state. The nuclei are said to be in resonance with external magnetic field. As the resonance frequencies and chemical shifts are unique or highly characteristic to individual compounds, NMR spectroscopy is powerful method for identification of small molecules in biological fluids such as in saliva (9). Further, as the area under a signal peak is proportional to the concentration of certain molecule, NMR spectroscopy allows quantitative analysis of salivary metabolites (14).

Identification of new salivary biomarkers would help us to diagnose HNSCC in its early stages, which is highly advantageous and can help in selecting the most appropriate treatment modalities. Here, we have used NMR spectroscopy to assess possible salivary metabolic changes associated with HNSCC. The aim was to compare the salivary metabolic profile between HNSCC patients and healthy controls.

\section{Patients and methods}

Patients and collection of saliva samples. A total of 45 consecutive patients with HNSCC were recruited to the longitudinal case control clinical study. The investigation was conducted in accordance with the ethical standards and according to the Declaration of Helsinki. The present study was approved by the Ethics Committee for Human Studies, Piracicaba Dental School, State University of Campinas, Sao Paulo Brazil (protocol no. 142/2010) and written informed consent was obtained from every participant. Patients' demographic and clinicopathologic data has been previously described by González-Arriagada et al (15). For this study, the collection of saliva samples from all patients was performed after dental treatment prior to radiotherapy. Unstimulated whole-mouth saliva sample was collected from all patients and from 30 healthy, non-smoking subjects (control group) in the morning, between 9 and 11 a.m., using standardized techniques (16). Each subject was asked to let the naturally produced saliva drain into a sterile glass cup for a period of $5 \mathrm{~min}$. The collected samples were then centrifuged $(14,000 \mathrm{rpm}$ for $6 \mathrm{~min})$. The supernatants were stored at $-20^{\circ} \mathrm{C}$ for subsequent NMR analysis.

Sample preparation. To each $450 \mu \mathrm{l}$ of saliva sample, $50 \mu \mathrm{l}$ of NMR-buffer (1.5 $\mathrm{M} \mathrm{KH}_{2} \mathrm{PO}_{4}, 2 \mathrm{mM} \mathrm{NaN}_{3}, 5.8 \mathrm{mM}$ sodium 3-(trimethylsilyl)propionate-2,2,3,3- $\mathrm{d}_{4}, \mathrm{D}_{2} \mathrm{O}, \mathrm{pH} 7.4$ ) was added and then the mixture was centrifuged at $10,000 \mathrm{~g}$ for $5 \mathrm{~min}$ at $4^{\circ} \mathrm{C}$ to remove any solid debris. The obtained supernatant was then transferred to NMR tubes (O.D. $5 \mathrm{~mm}$ ).
Data acquisition. NMR spectra were acquired using a 600.20 MHz Bruker AVANCE III HD spectrometer, equipped with a highly sensitive inverse triple resonance cryoprobe (Bruker CryoProbe Prodigy; Bruker BioSpin GmbH, Rheinstetten, Germany). The spectrometer was controlled via TopSpin 3.2 (Bruker BioSpin $\mathrm{GmbH}$ ) software. An automated shimming method (Topshim; Bruker BioSpin $\mathrm{GmbH}$ ) was used for all saliva samples which were preheated to $25^{\circ} \mathrm{C}$ about $30 \mathrm{~min}$ before the measurement. NMR data were acquired by employing a T2-relaxation-filtered pulse sequence that suppresses signals from macromolecule signals. In order to suppress the water peak, a Bruker cpmgld pulse sequence with $\mathrm{T}_{2}$-filter time of $80 \mathrm{msec}$ and irradiation field of $50 \mathrm{~Hz}$ was used. For each sample, the $90^{\circ}$ pulse was automatically calibrated. A receiver gain setting was kept as constant for all the samples.

Data processing. Samples were analysed blinded, in a random order. The raw NMR spectra were manually corrected for phase using TopSpin 3.0 software (Bruker BioSpin GmbH). A line-broadening factor of $1 \mathrm{~Hz}$ was applied to measured free induction decays prior to Fourier transformations. In total of 24 metabolites were identified by referring to the published literature $(17,18)$. The total-line-shape fitting tool in PERCH NMR software (PERCH Solutions Ltd, Kuopio, Finland) was used in the quantification of the metabolites. This method allows accurate quantification of identified metabolites even if the baseline is not linear or signals overlap (19). All the spectra were referenced to reference compound [(trimethylsilylpropanoic acid, (TSP)], used as an internal standard. The final metabolite concentrations are reported as $\mu \mathrm{mol} / 1$ in saliva.

Statistical analysis. Data are reported as the median and interquartile range (IQR). The distribution of metabolite concentration values was tested for normality using the Shapiro-Wilk test and the values of kurtosis and skewness. Salivary metabolite concentrations between HNSCC patients and healthy controls were compared with a non-parametric Mann-Whitney U-test. Collinearity between salivary metabolite pairs was assessed by computing the Pearson correlation matrix. $\mathrm{P}<0.05$ was considered to indicate a statistically significant difference. Multivariate discrimination function analysis (DFA) was employed to clarify which metabolites, when considering together, give maximum discrimination power between the groups. In the DFA, stepwise method with Wilk's lambda criterion was used. Two separate discriminant analyses were performed: i) Initially all salivary metabolites having no more than one missing value were entered; and ii) only those metabolites with no significant correlation with the best single predictor were entered. The limit for significant correlation coefficient was set to 0.50 . Finally, sensitivity and specificity of proposed discrimination model was determined. SPSS software, version 23.0 (IBM Corp., Armonk, NY, USA) was employed in all statistical analyses.

\section{Results}

Saliva samples collected from eight male patients with HNSCC, with a mean age of 61.7 \pm 9.6 years (range, 52-76 years), and from 30 controls, with a mean age of $54.4 \pm 9.0$ years (range 42-74 years) were included in the present study. There was 
Table I. Clinical characteristics of the 8 HNSCC patients analysed.

\begin{tabular}{|c|c|c|c|c|c|c|c|}
\hline Patient no. & $\begin{array}{c}\text { Age } \\
\text { (years) }\end{array}$ & Sex & $\begin{array}{c}\text { Tumour } \\
\text { localization }\end{array}$ & Stage & Smoking & Drinking & Hyposalivation \\
\hline 1 & 57 & Male & Larynx & I & Yes & Yes & No \\
\hline 14 & 56 & Male & Oral cavity & III & Yes & Yes & No \\
\hline 33 & 52 & Male & Larynx & IV & No & No & Mild \\
\hline 38 & 73 & Male & Oral cavity & III & Yes & Yes & Severe \\
\hline 42 & 65 & Male & Larynx & IV & Yes & Yes & Severe \\
\hline 43 & 57 & Male & Larynx & IV & Yes & Yes & No \\
\hline 44 & 76 & Male & Oral cavity & IV & Yes & Yes & Severe \\
\hline 45 & 53 & Male & Larynx & III & Yes & Yes & No \\
\hline
\end{tabular}

HNSCC, head and neck squamous cell carcinoma.

Table II. Comparison of salivary metabolite concentrations between patients with HNSCC $(n=8)$ and healthy controls ( $\mathrm{n}=30)$.

\begin{tabular}{|c|c|c|c|}
\hline Metabolite & HNSCC patients & Controls & P-value \\
\hline Butyrate & $74.2(33.9-266.4)$ & $58.6(25.9-128.4)$ & 0.562 \\
\hline Propionate & $659.0(319.9-2,157.6)$ & $527.3(251.1-1,028.4)$ & 0.428 \\
\hline 1,2-propanediol & $69.6(32.7-2,465.4)$ & $30.1(21.7-54.1)$ & $0.032^{\mathrm{a}}$ \\
\hline Fucose & $694.0(302.0-1,527.2)$ & $189.1(100.6-284.7)$ & $0.003^{b}$ \\
\hline Lactate & $207.5(71.5-1,132.9)$ & $197.4(140.4-324.6)$ & 0.986 \\
\hline Alanine & $90.3(47.1-515.9)$ & $107.4(53.0-173.0)$ & 0.820 \\
\hline Butanol & $59.9(17.2-190.5)$ & $36.5(16.8-84.3)$ & 0.428 \\
\hline Acetate & $2916.1(2,559.8-9,344.8)$ & $3282.4(1,977.7-5,239.5)$ & 0.428 \\
\hline Pyruvate & $27.3(12.6-73.1)$ & $13.9(7.2-33.3)$ & 0.148 \\
\hline Succinate & $50.6(24.5-214.3)$ & $58.9(47.1-71.9)$ & 0.765 \\
\hline Methylamine & $5.7(1.7-66.6)$ & $3.7(1.9-5.7)$ & 0.445 \\
\hline Choline & $17.1(12.2-43.7)$ & $19.2(14.2-24.7)$ & 0.765 \\
\hline Taurine & $133.8(72.1-195.4)$ & $170.2(104.7-205.1)$ & 0.502 \\
\hline Methanol & $118.0(36.6-208.1)$ & $80.4(51.4-121.5)$ & 0.515 \\
\hline Proline & $156.9(104.1-799.9)$ & $610.1(318.5-1,244.3)$ & $0.043^{\mathrm{a}}$ \\
\hline Tyrosine & $113.8(42.3-173.5)$ & $96.8(55.3-165.5)$ & 0.847 \\
\hline Phenylalanine & $100.9(41.9-147.6)$ & 79.7 (59.1-123.6) & 0.880 \\
\hline Formate & $229.7(191.4-426.2)$ & $178.3(77.0-433.3)$ & 0.428 \\
\hline Glycine & $560.8(103.2-719.1)$ & $494.3(241.1-923.6)$ & 0.582 \\
\hline
\end{tabular}

Data are expressed as the median (inter-quartile range). P-values are based on Mann-Whitney $\mathrm{U}$ test. ${ }^{\mathrm{a}} \mathrm{P}<0.05 ;{ }^{\mathrm{b}} \mathrm{P}<0.01$. HNSCC, head and neck squamous cell carcinoma.

no significant difference between the groups with respect to age $(\mathrm{P}=0.065)$. The reason for high number of rejected patient samples (37/45) was their limited sample volume, being too small for reliable NMR analysis. Out of $8 \mathrm{HNSCC}$ patients, primary tumour was located in the larynx in five patients and in oral cavity in three patients (Table I). All of the patients, except one, were diagnosed with advanced stage (III/IV) disease (Table I).

From each sample, up to 19 metabolites including organic acids (acetate, butyrate, formate, lactate, propionate, pyruvate, succinate), carbohydrates (1,2-propanediol, butanol, fucose, methanol), amino acids (alanine, glycine, phenylalanine, taurine, tyrosine) and amines (choline, methylamine, proline) were successfully quantified (Table II). Some metabolites, e.g., citrate, were detected only in some cases, and thus they were omitted in further analyses. In univariate analysis, the median concentrations of fucose and 1,2-propanediol were significantly higher $(\mathrm{P}=0.003, \mathrm{P}=0.032$, respectively) in the HNSCC patients compared to the controls. Instead, the proline was significantly lower $(\mathrm{P}=0.043)$ in the HNSCC saliva samples compared to controls. In respect of other metabolites, no statistically significant differences were observed.

The first stepwise DFA (in which all salivary metabolites having no more than one missing value were included) resulted 
in four salivary metabolites (fucose, glycine, methanol and proline) that being considered together results the maximal discriminating power between the two groups. The second DFA, when only those metabolites with no significant correlation with the best single predictor (i.e., fucose) were entered, resulted in just identical combination of metabolites. $92.1 \%$ of the originally grouped cases were correctly classified. A sensitivity of $87.5 \%$ (i.e., $7 / 8$ cancerous cases were correctly predicted) and specificity of $93.3 \%$ (i.e., 28/30 healthy cases were correctly predicted) was resulted. When only two metabolites (among the group of fucose, glycine, methanol and proline) were entered into the DFA, a pair of fucose-proline resulted in the highest discriminant power (90.9\%) (Fig. 1).

\section{Discussion}

The analysis of salivary metabolomic profile can offer an early phase approach to assess the changes associated with a wide range of diseases (8-10). Mass spectrometry (MS) and NMR are the most common analytical techniques used in metabolomics. The two techniques have distinct advantages and limitations. Compared to NMR, MS is significantly more sensitive to identify broader variety of the metabolites (18). However, MS analyses of complex biofluids requires a metabolite separation prior to measurement, and thus MS is rarely used alone but usually coupled to separation techniques like gas chromatography (GC-MS) or liquid chromatography (LC-MS). This may bias any analysis. Furthermore, the quantification by MS is challenging. The main benefits of NMR spectroscopy include its minimal sample handling, unbiased quantification of low molecular weight compounds and high reproducibility (17-19). In this study, we assessed salivary metabolic alterations associated with HNSCC by NMR spectroscopy. Our study showed that NMR analysis is a robust approach to study the metabolome of saliva that is sensitive to metabolic changes in HNSCC. In a univariate analysis, two metabolites, i.e., fucose and 1,2-propanediol were significantly upregulated, whereas proline was significantly downregulated in HNSCC. However, in a multivariate analysis, a combination of four salivary metabolites (fucose, glycine, methanol and proline) together provided maximum discrimination among HNSCC patients and healthy controls.

6-deoxy-L-galactose (fucose) is a monosaccharide and an important constituent of glycoproteins. Fucosylation of glycoproteins, i.e., a process of adding fucose units at the terminal end of the oligosaccharide chain mediates several specific biologic functions (20) and known to occur during the development of cancer. Tumour cells modulate their surface by increasing fucosylation levels that leads to several abnormal cellular characteristics, such as decreased adhesion and uncontrolled tumour growth (21). In normal tissues, fucosylation levels are relatively low, but rapidly increases during carcinogenesis. Therefore, several researchers have speculated that the monitoring of serum fucose levels could be a potential approach for the early detection, diagnosis, and prognosis of cancers (22-25). It has been suggested that the increased presence of fucose is caused more by local synthesis by tumour cells than destruction of the malignant cells $(26,27)$. Shah et al (23) analysed blood samples collected from 130 patients with untreated oral cancer (OC), from 75 patients with oral precancerous conditions and from 100 healthy controls. They

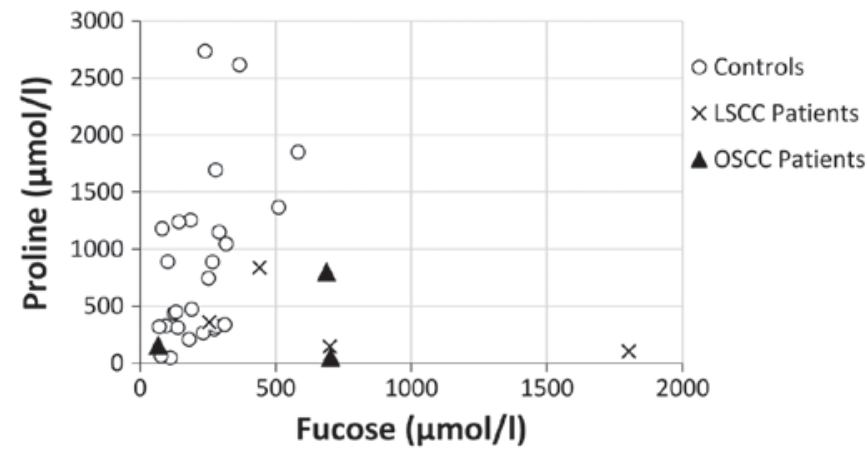

Figure 1. Scatter plot presenting the salivary proline concentration $(\mu \mathrm{mol} / \mathrm{l})$ against salivary fucose concentration $(\mu \mathrm{mol} / \mathrm{l})$ for each patient and the control subjects. LSCC, laryngeal squamous cell carcinoma; OSCC, oral squamous cell carcinoma; fucose, 6-deoxy-L-galactose.

found that serum fucose levels were significantly elevated in OC patients OC compared with patients having oral precancerous lesions or healthy controls. Shetty et al (28) estimated serum L-fucose glycoprotein levels among 50 HNSCC patients in comparison of 50 age- and sex-matched healthy controls. They reported over 2-fold increase in serum glycoprotein L-fucose in patients compared to controls. Findings reported by other researchers are also consistent $(29,30)$. However, according to our best knowledge, this study, for the first time, demonstrates that HNSCC-induced alterations in fucose levels can be also detected in unstimulated whole-mouth saliva.

Previous saliva-based studies aimed to monitor aberrant glucosylation in cancer diagnostics have focused on sialic acid (N-acetyl neuraminic acid) that is a negatively charged nine-carbon monosaccharide. Sialic acids are also important terminal sugars in cell membrane glycoproteins and glycolipids (31). Previous studies have showed elevated levels of serum and salivary sialic acid in various carcinomas, including oral pre-cancer and OC (32-37). Unfortunately, sialic acid was not among these 19 metabolites that we managed to identify and quantify in the present study. Recently, Dame et al (18) identified and quantified by ${ }^{1} \mathrm{H}-\mathrm{NMR}$ a total of 76 different metabolites from saliva samples including short chain organic acids, amino acids, alcohols, amines, sugars and pharmaceutical adjuvants. Among these metabolites, 41 were unique, i.e., they were not detected by other metabolomics methods. In contrast, some compounds were detected by GC-MS or LC-MS but not by NMR. These compounds either do not consist of NMR-detectable protons or their concentrations were below the detection limit (18).

Altered serum proline levels are known to be related to cancer metabolism. In previous studies, significantly descended levels of proline have been observed in serum samples taken from patients suffering from renal cell carcinoma (38), oral cancer (39) and esophageal cancer (40). The lowered proline level is expected to be an indicator of overutilization of amino acids in the tumor tissue (40). We consistently observed significantly lowered salivary proline levels in patients with HNSCC in comparison to healthy individuals. Interestingly, salivary proline concentration against fucose concentration seems to provide a promising linear discrimination power for HNSCC, even though present study is limited due to the low number of patient samples. We had to reject major part (82\%) of patient samples due to the limited sample volume that was not adequate 
for reliable NMR analysis. NMR analysis requires a relatively large saliva sample $(\sim 0.5 \mathrm{ml})$ that may be challenging when collecting unstimulated saliva, especially patients with dry mouth. NMR experiments were not originally planned to this patient population and thus sufficient sample volume collection was not systematically ensured. Furthermore, this study consists of saliva samples taken from patients whose primary tumour was located either in the larynx or in oral cavity. We acknowledge that the etiology of these diseases differ in their etiopathogenesis. However, whole saliva is a complex biofluid deriving from the secretion of salivary glands, gingival folds and oral mucosal transudate. In addition, it includes exudates from mucous of the nasal cavity and pharynx, blood cells, bacterial metabolites, food remainders, desquamated epithelial cells, traces of medications or chemical products (41). Therefore, it is rational that also laryngeal squamous cell carcinoma origin alterations can be found in saliva.

As a diagnostic media, saliva fulfills essential criteria such as an ease and non-invasive collection and low-cost handling and storage of samples. Saliva consists of a numerous compounds such as proteins, peptides, nucleic acids, electrolytes, and hormones originating from multiple local and systemic sources that can be used as disease specific biomarkers in diagnosis and disease monitoring (41). Unlike blood, saliva does not clot and saliva analytes are stable and cost-efficient to store. Problems of low concentration of relevant biomarker compounds in saliva have been largely surpassed through several instrumental and analytical advancements in the field of omics technologies (42). Furthermore, pain, anxiety and infection risk closely related to traditional methods, i.e., blood collection or tissue biopsy can be avoided in saliva-based diagnostics. Feasibility of multiple repetition sampling is also a significant bonus for disease screening, diagnostics and follow-up of treatment and rehabilitation outcomes. All in all, the collection, processing and analysis of saliva can be considered as easier than corresponding procedures for blood or any other biological fluid (43).

Besides oral diseases, salivary analysis is highly potential also in diagnostics of various systemic diseases like Sjögren's syndrome $(44,45)$ as well as distant malignancies, such as breast cancer $(46)$, lung cancer $(47,48)$ and pancreatic cancer $(49,50)$. Although saliva is able to reflect well the overall health, its use as a diagnostic media is still rare. The current evidence about the diagnostic potential of reported salivary biomarkers in various pathologies is still weak and needs to be strengthen in further validation studies with larger number of samples. Further studies with various state-of-the-art 'omics' methods can help in developing a prospective disease-specific biomarker pattern based on these molecules (51). It is evident that metabolic map of cancer contains more than one biomarker molecule and therefore, salivary metabolomics based on NMR or MS is a useful quantitative technique to screen wide variety of salivary components (18). Identification of reliable, disease-specific biomarkers can allow the development of novel point-of-care (POC) platforms enabling simple and cost-effective quantification of target biomarker molecules. Incorporating these POC approaches into the part of primary health screening programs, the burden on health care sector in terms of costly equipment and invasive testing procedures can be significantly reduced in the future $(3,6,43)$. Although this study provides promising preliminary results, controlled longitudinal trials with higher number of patients are needed to ensure the true diagnostic accuracy and feasibility to build up a real diagnostic saliva test. Moreover, these biomarkers need to be further examined in other aspects of HNSCC such as monitoring of therapy response and classification of disease severity.

\section{Acknowledgements}

The authors acknowledge the contribution of NMR Metabolomics Laboratory at the University of Eastern Finland (Kuopio, Finland).

\section{Funding}

The present study was supported by the Finnish Funding Agency for Technology and Innovation (Tekes) project 'Novel spectroscopic methods for early detection and screening of oral cancer' (grant no. 52/31/2014).

\section{Availability of data and materials}

The datasets used and/or analyzed during the current study are available from the corresponding author on reasonable request.

\section{Authors' contributions}

TS, WAGA, MAL, AMK and SM designed the study. JJWM, RA, WAGA, MAL, AMK and SM performed the experiments and acquired the data. JJWM, SPS, RA, RL, AMK and SM analyzed and interpreted the data. JJWM, SPS and SM were the major contributors in writing the manuscript. All authors critically reviewed and approved the final manuscript.

\section{Ethics approval and consent to participate}

The investigation was conducted in accordance with ethical standards and according to the Declaration of Helsinki. The present study was approved by the Ethics Committee for Human Studies, Piracicaba Dental School, State University of Campinas, Brazil (protocol no. 142/2010) and written informed consent was obtained from every participant.

\section{Patient consent for publication}

Not applicable.

\section{Competing interests}

The authors declare that they have no competing interests.

\section{References}

1. Ferlay J, Soerjomataram I, Dikshit R, Eser S, Mathers C, Rebelo M, Parkin DM, Forman D and Bray F: Cancer incidence and mortality worldwide: Sources, methods and major patterns in GLOBOCAN 2012. Int J Cancer 136: E359-E386, 2015.

2. Abbey LM, Kaugars GE, Gunsolley JC, Burns JC, Page DG, Svirsky JA, Eisenberg E, Krutchkoff DJ and Cushing M: Intraexaminer and interexaminer reliability in the diagnosis of oral epithelial dysplasia. Oral Surg Oral Med Oral Pathol Oral Radiol Endod 80: 188-191, 1995.

3. Liu J and Duan Y: Saliva: A potential media for disease diagnostics and monitoring. Oral Oncol 48: 569-577, 2012. 
4. Zhang CZ, Cheng XQ, Li JY, Zhang P, Yi P, Xu X and Zhou: Saliva in the diagnosis of diseases. Int J Oral Sci 8: 133-137, 2016

5. Yoshizawa JM, Schafer CA, Schafer JJ, Farrell JJ, Paster BJ and Wong DT: Salivary biomarkers: Toward future clinical and diagnostic utilities. Clin Microbiol Rev 26: 781-791, 2013.

6. Cuevas-Córdoba B and Santiago-García J: Saliva: A fluid of study for OMICS. OMICS 18: 87-97, 2014.

7. Guerra EN, Acevedo AC, Leite AF, Gozal D, Chardin H and De Luca Canto G: Diagnostic capability of salivary biomarkers in the assessment of head and neck cancer: A systematic review and meta-analysis. Oral Oncol 51: 805-818, 2015.

8. Patel S and Ahmed S: Emerging field of metabolomics: Big promise for cancer biomarker identification and drug discovery. J Pharm Biomed Anal 107: 63-74, 2015.

9. Mikkonen JJW, Herrala M, Soininen P, Lappalainen R, Tjäderhane L, Seitsalo H, Niemelä R, Salo T, Kullaa A and Myllymaa S: Metabolic profiling of saliva in patients with primary Sjögren's syndrome. Metabolomics 3: 128, 2013.

10. Spielmann N and Wong DT: Saliva: Diagnostics and therapeutic perspectives. Oral Dis 17: 345-354, 2011.

11. Bonne NJ and Wong DT: Salivary biomarker development using genomic, proteomic and metabolomic approaches. Genome Med 4: 82, 2012

12. Winck FV, Prado Ribeiro AC, Ramos Domingues R, Ling LY, Riaño-Pachón DM, Rivera C, Brandão TB, Gouvea AF, Santos-Silva AR, Coletta RD and Paes Leme AF: Insights into immune responses in oral cancer through proteomic analysis of saliva and salivary extracellular vesicles. Sci Rep 5: 16305, 2015

13. Zlotogorski-Hurvitz A, Dayan D, Chaushu G, Salo T and Vered M: Morphological and molecular features of oral fluid-derived exosomes: Oral cancer patients versus healthy individuals. J Cancer Res Clin Oncol 142: 101-110, 2016.

14. Günther H: NMR spectroscopy: Basic principles, concepts, and applications in chemistry, 2 edition, John Wiley and Sons Ltd., Chichester, 1995.

15. González-Arriagada WA, Ramos LM, Silva AA, Vargas PA Coletta RD, Bingle L and Lopes MA: Salivary BPIFA1 (SPLUNC1) and BPIFA2 (SPLUNC2 A) are modified by head and neck cancer radiotherapy. Oral Surg Oral Med Oral Pathol Oral Radiol 119: 48-58, 2015

16. Navazesh M: Methods for collecting saliva. Ann N Y Acad Sci 694: 72-77, 1993.

17. Silwood CJ, Lynch E, Claxson AW and Grootveld MC: $1 \mathrm{H}$ and (13)C NMR spectroscopic analysis of human saliva. J Dent Res 81: 422-427, 2002.

18. Dame ZT, Aziat F, Mandal RS, Krishnamurthy R, Bouatra S, Borzouie S, Guo AC, Sajed T, Deng L, Lin H, et al: The human saliva metabolome. Metabolomics 11: 1864-1883, 2015.

19. Soininen P, Haarala J, Vepsäläinen J, Niemitz $M$ and Laatikainen R: Strategies for organic impurity quantification by $1 \mathrm{H}$ NMR spectroscopy: Constrained total-line-shape fitting. Anal Chim Acta 542: 178-185, 2005

20. Ma B, Simala-Grant JL and Taylor DE: Fucosylation in prokaryotes and eukaryotes. Glycobiology 16: 158R-184R, 2006

21. Miyoshi E, Moriwaki K and Nakagawa T: Biological function of fucosylation in cancer biology. J Biochem 143: 725-729, 2008.

22. Fernández-Rodríguez J, Páez de la Cadena M, Martínez-Zorzano VS and Rodríguez-Berrocal FJ: Fucose levels in sera and in tumours of colorectal adenocarcinoma patients. Cancer Lett 121: 147-153, 1997.

23. Shah M, Telang S, Raval G, Shah P and Patel PS: Serum fucosylation changes in oral cancer and oral precancerous conditions: Alpha-L-fucosidase as a marker. Cancer 113: 336-346, 2008.

24. Manjula S, Monteiro F, Rao Aroor A, Rao S, Annaswamy R and Rao A: Assessment of serum L-fucose in brain tumor cases. Ann Indian Acad Neurol 13: 33-36, 2010.

25. Listinsky JJ, Siegal GP and Listinsky CM: The emerging importance of $\alpha$-L-fucose in human breast cancer: A review. Am J Transl Res 3: 292-322, 2011.

26. Shetlar MR, Foster JV, Kelly KH, Shetlar CL, Bryan RS and Everett MR: The serum polysaccharide level in malignancy and in other pathological conditions. Cancer Res 9: 515-519, 1949.

27. Sawke NG and Sawke GK: Serum fucose level in malignant diseases. Indian J Cancer 47: 452-457, 2010.

28. Shetty RK, Bhandary SK and Kali A: Significance of serum L-fucose glycoprotein as cancer biomarker in head and neck malignancies without distant metastasis. J Clin Diagn Res 7: 2818-2820, 2013.

29. Parwani RN and Parwani SR: Quantitative evaluation of serum fucose in oral squamous cell carcinoma patients. J Can Res Ther 7: 143-147, 2011.
30. Rai NP, Anekar J, Shivaraja Shankara YM, Divakar DD, Al Kheraif AA, Ramakrishnaiah R, Sebastian R, Raj AC, Al-Hazmi A and Mustafa SM: Comparison of serum fucose levels in leukoplakia and oral cancer patients. Asian Pac J Cancer Prev 16: 7497-7500, 2015.

31. Chugh S, Gnanapragassam VS, Jain M, Rachagani S, Ponnusamy MP and Batra SK: Pathobiological implications of mucin glycans in cancer: Sweet poison and novel targets. Biochim Biophys Acta 1856: 211-225, 2015.

32. Rajpura KB, Patel PS, Chawda JG and Shah RM: Clinical significance of total and lipid bound sialic acid levels in oral pre-cancerous conditions and oral cancer. J Oral Pathol Med 34: 263-267, 2005

33. Sanjay PR, Hallikeri K and Shivashankara AR: Evaluation of salivary sialic acid, total protein, and total sugar in oral cancer: A preliminary report. Indian J Dent Res 19: 288-291, 2008.

34. Sawhey H and Kumar CA: Correlation of serum biomarkers (TSA \& LSA) and epithelial dysplasia in early diagnosis of oral precancer and oral cancer. Cancer Biomark 10: 43-49, 2011

35. Taqi SA: Clinical evaluation of total and lipid bound sialic acid levels in oral precancer and oral cancer. Indian J Med Paediatr Oncol 33: 36-41, 2012.

36. Dhakar N, Astekar M, Jain M, Saawarn S and Saawarn N: Total sialic acid, total protein and total sugar levels in serum and saliva of oral squamous cell carcinoma patients: A case control study. Dent Res J (Isfahan) 10: 343-347, 2013.

37. Dadhich M, Prabhu V, Pai VR, D'Souza J, Harish S and Jose M: Serum and salivary sialic acid as a biomarker in oral potentially malignant disorders and oral cancer. Indian J Cancer 51: 214-218, 2014 .

38. Mustafa A, Gupta S, Hudes GR, Egleston BL, Uzzo RG and Kruger WD: Serum amino acid levels as a biomarker for renal cell carcinoma. J Urol 186: 1206-1212, 2011.

39. Tiziani S, Lopes V and Günther UL: Early stage diagnosis of oral cancer using $1 \mathrm{H}$ NMR-based metabolomics. Neoplasia 11: 269-276, 4p, 2009.

40. Liang S, Sanchez-Espiridion B, Xie H, Ma J, Wu X and Liang D: Determination of proline in human serum by a robust LC-MS/MS method: Application to identification of human metabolites as candidate biomarkers for esophageal cancer early detection and risk stratification. Biomed Chromatogr 29: 570-577, 2015.

41. Humphrey SP and Williamson RT: A review of saliva: Normal composition, flow, and function. J Prosthet Dent 85: 162-169, 2001.

42. Tiwari M: Science behind human saliva. J Nat Sci Biol Med 2. 53-58, 2011.

43. Koneru S and Tanikonda R: Salivaomics-A promising future in early diagnosis of dental diseases. Dent Res J (Isfahan) 11: 11-15, 2014.

44. Hu S, Gao K, Pollard R, Arellano-Garcia M, Zhou H, Zhang L, Elashoff D, Kallenberg CG, Vissink A and Wong DT: Preclinical validation of salivary biomarkers for primary Sjögren's syndrome. Arthritis Care Res (Hoboken) 62: 1633-1638, 2010.

45. Mikkonen JJ, Singh SP, Herrala M, Lappalainen R, Myllymaa S and Kullaa AM: Salivary metabolomics in the diagnosis of oral cancer and periodontal diseases. J Periodontal Res 51: 431-437, 2016.

46. Bigler LR, Streckfus CF, Copeland L, Burns R, Dai X, Kuhn M, Martin P and Bigler SA: The potential use of saliva to detect recurrence of disease in women with breast carcinoma. J Oral Pathol Med 31: 421-431, 2002.

47. Zhang L, Xiao H, Zhou H, Santiago S, Lee JM, Garon EB, Yang J, Brinkmann O, Yan X, Akin D, et al: Development of transcriptomic biomarker signature in human saliva to detect lung cancer. Cell Mol Life Sci 69: 3341-3350, 2012.

48. Xiao H, Zhang L, Zhou H, Lee JM, Garon EB and Wong DT: Proteomic analysis of human saliva from lung cancer patients using two-dimensional difference gel electrophoresis and mass spectrometry. Mol Cell Proteomics 11: M111.012112, 2012.

49. Sugimoto M, Wong DT, Hirayama A, Soga T and Tomita M: Capillary electrophoresis mass spectrometry-based saliva metabolomics identified oral, breast and pancreatic cancer-specific profiles. Metabolomics 6: 78-95, 2010.

50. Lau C, Kim Y, Chia D, Spielmann N, Eibl G, Elashoff D, Wei F, Lin YL, Moro A, Grogan T, et al: Role of pancreatic cancer-derived exosomes in salivary biomarker development. J Biol Chem 288: 26888-26897, 2013.

51. Grant MM: What do 'omic technologies have to offer periodontal clinical practice in the future? J Periodontal Res 47: 2-14, 2012. 